\title{
ARTICLE
}

Cite this: DOI: $10.1039 /$ xoxxooooox

\section{Tailoring the edge of graphene quantum dots to establish localized $\pi-\pi$ interactions with aromatic molecules}

Received ooth January 2012,

Accepted ooth January 2012

DOI: $10.1039 /$ xoxxooooox

www.rsc.org/

\author{
Shujun Wang ${ }^{a}$, Zachary Lemon ${ }^{a}$, Ivan S. Cole ${ }^{b}$ and ${\operatorname{Qin~} \mathrm{Li}^{a}}^{*}$
}

We report a simple route combining bottom-up and top-down strategies for the synthesis of partially ring-terminated graphene quantum dots (GQDs) employing aniline for modifying the molecular structure of GQDs edges. In contrast to the GQDs synthesized without introducing ring-terminals by aniline conjugation, the photoluminescence of such partially ring-terminated GQDs can be quenched by the aromatic molecules owing to the edge-localized $\pi-\pi$ interactions. This study provides a generalizable strategy in functionalizing GQDs towards aromatic molecule sensors, as well as important insight to the effect of edge-localized $\pi-\pi$ interaction in perturbing the electronic structure of GQDs.

\section{Introduction,}

Graphene quantum dots (GQDs) have rapidly emerged as one of the important derivatives of graphene with unique optical properties and promising applications in optical and optoelectronics devices $^{1,2}$. Tuning the properties of GQDs is of great value to both fundamental understanding and practical applications of this new material. It has been shown by both theoretical and experimental works that in addition to size and shape $^{3-6}$, the edge of GQDs also plays a decisive role in their physical and chemical properties ${ }^{7-9}$. Chemical doping ${ }^{10-12}$ and functionalization $^{13,14}$ are effective means to modify GQDs edges.

Comprised of $\mathrm{sp}^{2}$ carbons and rich in $\pi$ electrons, GQDs are deemed to be an ideal platform to allow $\pi-\pi$ interactions to take place. Such $\pi-\pi$ interactions can result in charge or energy transfers, leading to changes in the photoluminescence ( PL) behaviour of GQDs ${ }^{15}$. Hence, GQDs are poised to be excellent optical sensing agents for aromatic molecules. However, so far most of the GQDs sensing works reported on ionic species $2,16,17$ and only limited literature ${ }^{18}$ have addressed the stimuli-response of GQDs to $\pi-\pi$ interactions with aromatic analytes. Such a under-utilization of the $\pi-\pi$ interactions in GQDs sensing may be due to the fact that: 1) little or no $\pi-\pi$ interactions could take place on the basal plane, or such $\pi-\pi$ interactions pose limited influence to the PL of GQDs; 2) the $\pi$ $\pi$ interactions at the edge may be inhibited due to the abundant oxygen functional groups and disordered structures (i.e. $\mathrm{sp}^{3}$ structures, defects) around the edge ${ }^{19,20}$. Therefore, we postulate that if $\pi-\pi$ interaction with aromatic molecules localized at the peripheral region of GQDs could be established, noticeable changes in the PL of GQDs may be induced.

Herein, we report a facile route combining bottom-up and top-down processes for synthesis of partially ring-terminated GQDs (prGQDs). In contrast to that of regular GQDs, the PL of prGQDs can be quenched by aromatic analytes arising from the establishment of edge-localized $\pi-\pi$ interactions. We will also demonstrate that the effects of such an edge functionalization on the optical properties and sensing selectivity of GQDs.

\section{Experimental}

\subsection{Bottom-up synthesis of carbon sheets}

In a typical experiment, $1 \mathrm{~g}$ of citric acid (Chem-supply) was added into a round flask which was then placed in a silicon oil bath at $270^{\circ} \mathrm{C}$. The flask was removed from the oil bath after $20 \mathrm{~min}$ of reaction when the colourless citric acid turned into dark brown. After the flask cooled down to room temperature, $10 \mathrm{ml}$ sodium hydroxide solution $(15 \mathrm{mg} / \mathrm{mL}$, Chem-supply) was added into the flask to obtain a brown dispersion. The $\mathrm{pH}$ value of the brown dispersion was subsequently tuned to 7 by slowly adding more sodium hydroxide solution. The neutralized dispersion was subject to dialysis for 2 days with a $3.5 \mathrm{KD}$ a tube membrane (Spectrum Labs) in abundant deionized water to remove the small particles and salts. The remaining brown carbon sheets (CS) dispersion ${ }^{17}$ in the membrane tube was collected for further experiment.

2.2 Hydrothermal synthesis of graphene quantum dots (GQDs) and partially ring-terminated GQDs (prGQDs)

The CS dispersion $(\sim 2.5 \mathrm{mg} / \mathrm{mL})$ from previous section was firstly diluted to $0.12 \mathrm{mg} / \mathrm{mL}$. $14 \mathrm{~mL}$ of the diluted CS dispersion was then added into an autoclave (the volume of the Teflon cell is $28 \mathrm{~mL}$ ). For GQDs synthesis, the autoclave was tightly sealed then placed into an oven overnight with temperature set at $200^{\circ} \mathrm{C}$. For prGQDs synthesis, $25.6 \mu \mathrm{L}$ (25mg) of aniline (99\%, Chem-Supply) was added into the Teflon cell in addition to the CS dispersion as aforementioned. The autoclave was then sealed up for hydrothermal treatment 
overnight in the oven at $200^{\circ} \mathrm{C}$. Upon completion of the hydrothermal treatment, the dispersion inside the autoclave was purified by a $500 \mathrm{Da}$ dialysis tube membrane (Spectrum Labs) for 2 days to remove any excessive aniline. Following the purification, another dialysis treatment with $10 \mathrm{~K}$ Da dialysis tube membrane (Spectrum Labs) was applied as a filtration process to remove any residual large CS particles.

\subsection{Characterization}

Fluorescence spectra were collected through a Fluorescence spectrometer (Thermo Scientific Lumina). An UV/Vis spectrometer (Agilent 8453) was employed to acquire the absorption spectra. Chemical bonding types for the as synthesized materials were qualitatively evaluated through a Fourier transformation infrared (FTIR) spectrometer (Spectrum Two, Perkin Elmer). Elemental compositions and chemical bonding status were further quantitatively analysed by X-ray photoelectron spectroscopy (XPS, Kratos Axis Ultra). High resolution transmission electron microscopy (HRTEM, Philips
Tecnai F20), along with atomic force microscope (AFM, NTMDT NTEGRA Spectra) were adopted for the morphology analysis.

\section{Results and discussion}

\subsection{As-synthesized CS, GQDs and prGQDs}

The GQDs were synthesized by a combined bottom-up and topdown approach in which CS flakes were first synthesized via thermal decomposition of citric $\operatorname{acid}^{17,}{ }^{21}$ and then hydrothermally cut into GQDs. prGQDs were obtained by introducing aniline into the reaction system of hydrothermal treatment.

Figure 1 presents the morphology of the as-synthesized CS, GQDs and prGQDs. The CS obtained from bottom-up route ${ }^{17}$ contains graphitic crystallites of $5 \sim 7 \mathrm{~nm}$ in size scattered within an amorphous network as shown in Figure 1a. This feature of crystallites being loosely held together by an amorphous network makes it amenable to release the crystallites through the energy shock of hydrothermal treatment, because the
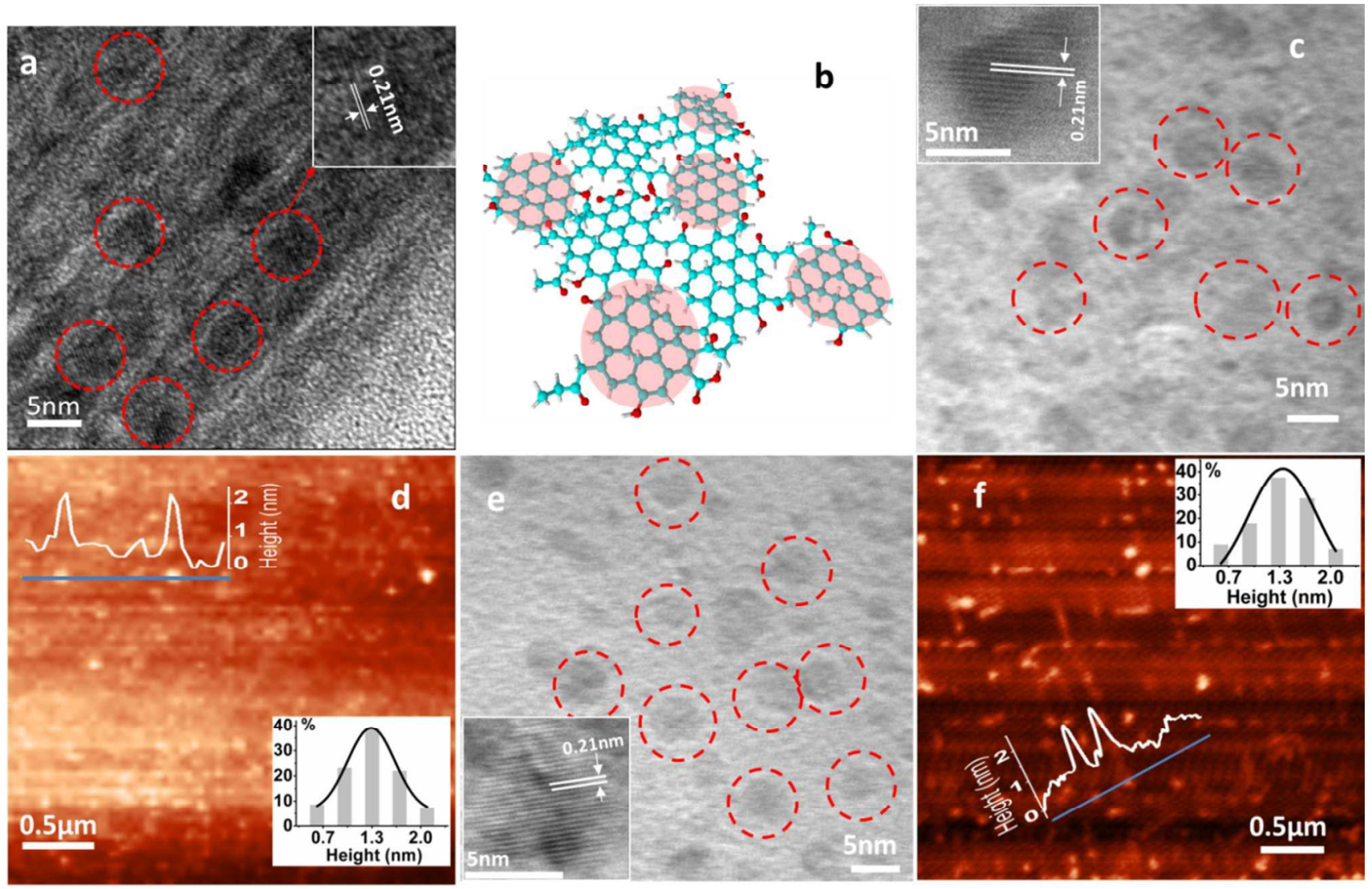

Figure 1. Morphology of CS, GQDs and prGQDs: (a), HRTEM image of CS showing graphitic domains ( $5 \mathrm{~nm})$ are scattered within amorphous structures; (b), Schematic representation of CS; (c) and (d), HRTEM image and AFM images of GQDs; e) and f), HRTEM image and AFM images of prGQDs. Inserts in $d$ and $f$ are the height profile of two dots along the strikethrough lines and, height distributions each of which based on over 300 dots.

amorphous network is structurally weaker than the graphitic domains. Once released, those $\mathrm{sp}^{2}$ crystallites become discrete GQDs. For synthesizing prGQDs, aniline was introduced in the hydrothermal cutting step, where the release of GQDs from the parent CS flakes and, functionalization by aniline occurred simultaneously. The aniline is attached on the edge of GQDs through the reactions between the carboxyl and hydroxyl groups on GQDs and the amine moiety of aniline. The high 
resolution transmission electron microscopy (HRTEM) images show that the sizes of crystallites within CS sheets, GQDs and prGQDs are all similar $(5 \sim 7 \mathrm{~nm})$, indicating the hydrothermal treatment is non-destructive to the crystallites. Moreover, the height profile statistics via atomic force microscope (AFM)

\subsection{Analysis of chemical composition}

The abundance of oxygen functional groups on the edge of GQDs renders them amenable to functionalization ${ }^{13,14}$. In our study, successful ring termination through esterification and
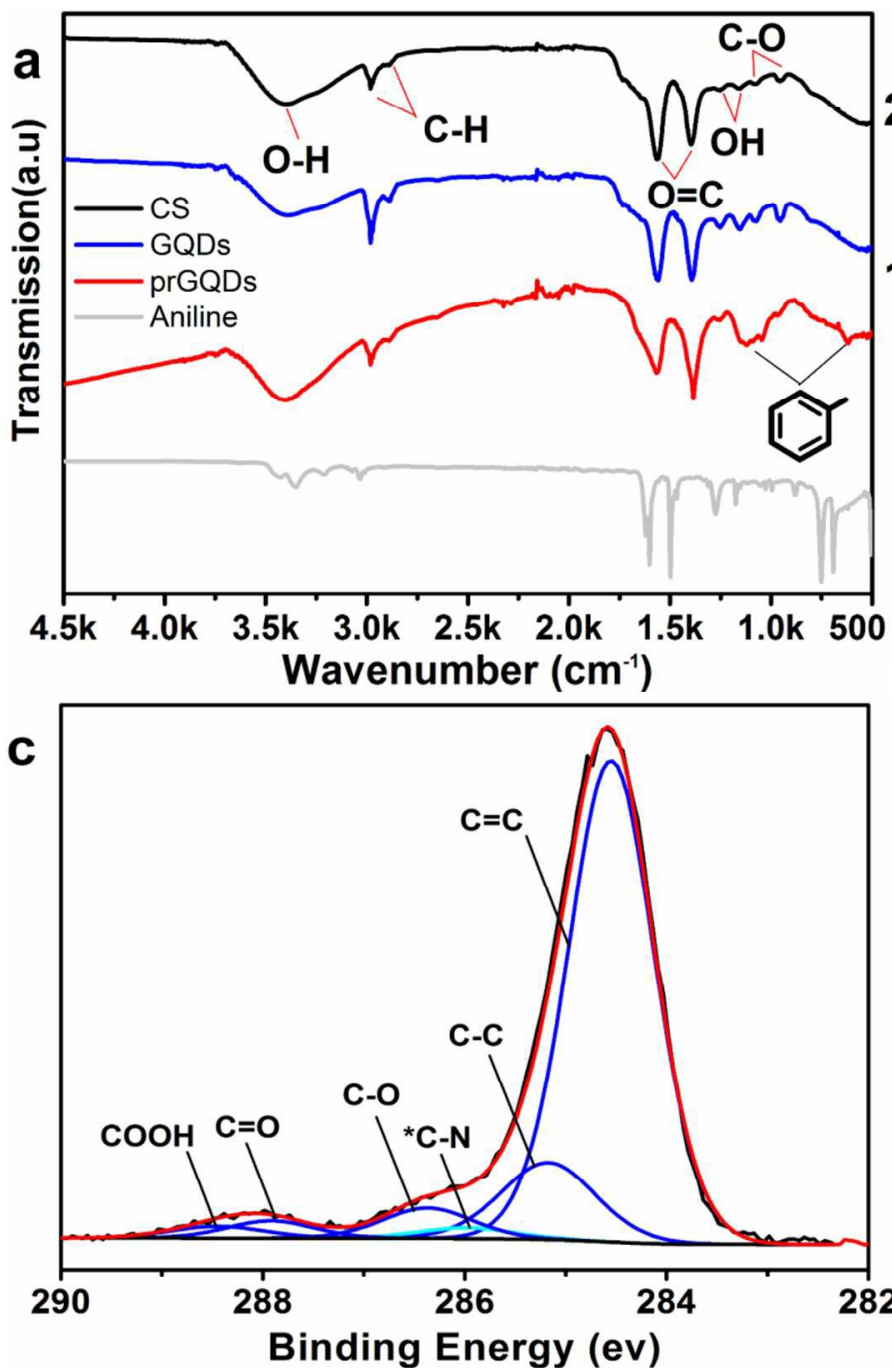

Figure 2. Chemical bonding analysis: (a), FTIR of CS, GQDs and prGQDs; (b) Summary on oxygen and nitrogen atomic concentration from XPS survey and (c); C1s XPS spectra of CS and prGQDs, respectively, (note: ${ }^{*} \mathrm{C}-\mathrm{N}$ in $\mathrm{c}$ represents both $\mathrm{O}=\mathrm{C}$ $\mathrm{N}$ and $\mathrm{C}-\mathrm{N})$; (d), N1s XPS spectrum of prGQDs

with various oxygen containing functional groups including two bands at $957 \mathrm{~cm}^{-1}$ and $1073 \mathrm{~cm}^{-1}$ due to the stretching of C$\mathrm{O}$, two bands at $1251 \mathrm{~cm}^{-1}$ and $1147 \mathrm{~cm}^{-1}$ as a result of $\mathrm{OH}$ bending, two bands at $1560 \mathrm{~cm}^{-1}$ and $1390 \mathrm{~cm}^{-1}$ caused by the $\mathrm{O}=\mathrm{C}$ stretching, two bands at $2878 \mathrm{~cm}^{-1}$ and $2982 \mathrm{~cm}^{-1}$ due to the $\mathrm{C}-\mathrm{H}$ stretching and one last band at $3387 \mathrm{~cm}^{-1}$ generated by $\mathrm{O}-\mathrm{H}$ stretching. In contrast, two new bands at $620 \mathrm{~cm}^{-1}$ and $1120 \mathrm{~cm}^{-1}$ emerged on the FTIR spectrum of prGQDs. The former is caused by the out plane bending of benzene ring, and the latter is due to the in plane stretching and bending of benzene ring. In addition, a comparison between normalized

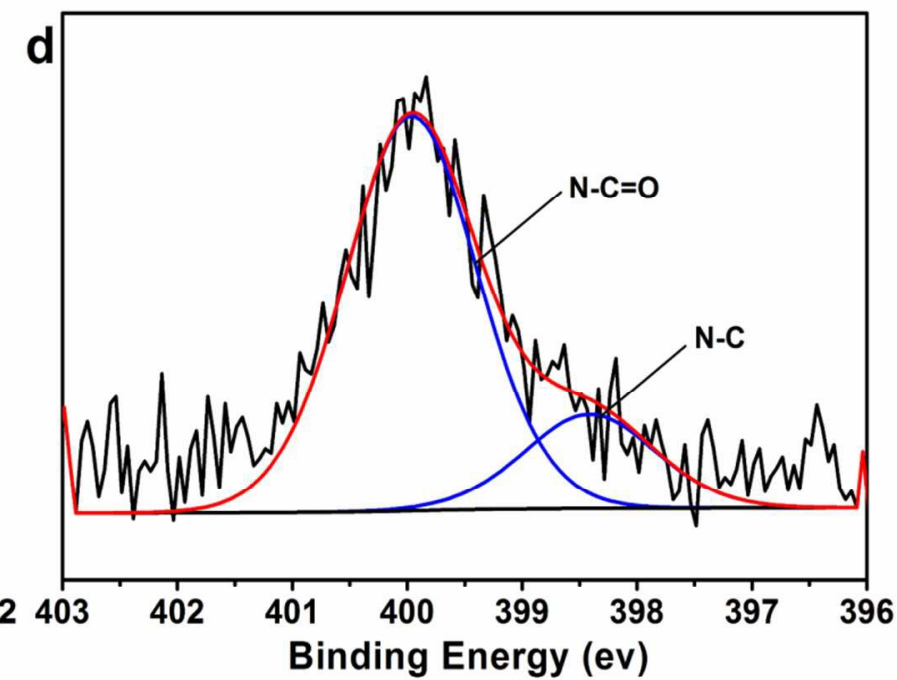

scans show that both GQDs and prGQDs possess a similar thickness of $\sim 1.4 \mathrm{~nm}$, which counts for 1 or 2 layers of carbon atoms.

amidation between aniline and GQDs are confirmed by both Fourier transform infrared spectra (FTIR) and X-ray photo spectroscopy (XPS). As shown in Figure 2a, CS and GQDs have almost identical FTIR spectra containing bands associated

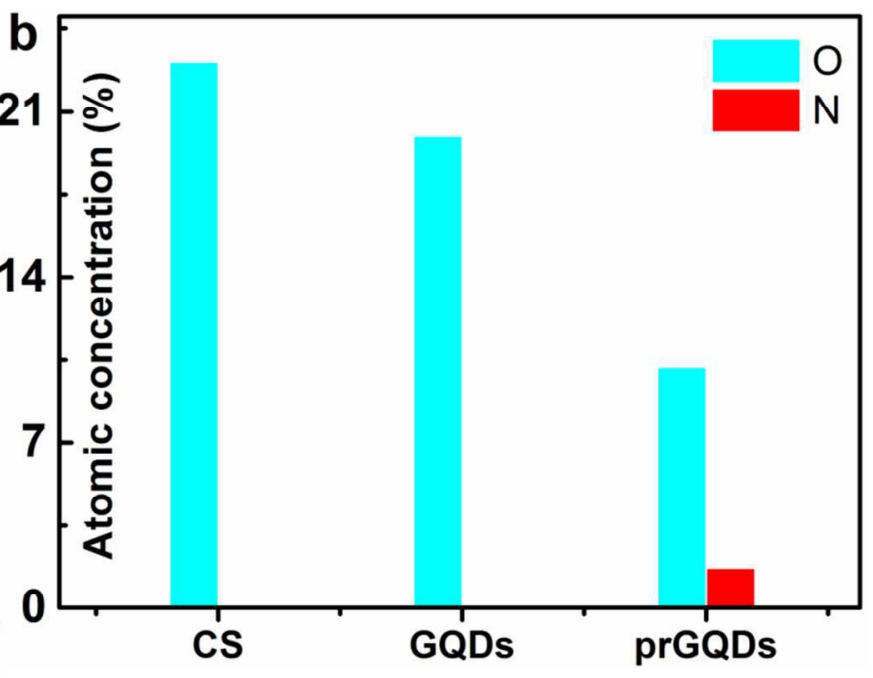

FTIR spectra of CS, GQDs and prGQDs within the range of $3000 \mathrm{~cm}^{-1}$ to $3750 \mathrm{~cm}^{-1}$, showed a blue shift of absorption peak of prGQDs with respect to CS and GQDs (Figure S2), which can be ascribed to the partial substitution of $-\mathrm{OH}$ stretching with $-\mathrm{NH}$ stretching due to the consumption of $-\mathrm{OH}$ upon aniline conjugation. The FTIR spectrum of pure aniline is also presented in Figure 2a for comparison. It could be drawn that the covalent bonding between aniline and GQDs demotes most of the aniline bands except for the 2 bands at $1120 \mathrm{~cm}^{-1}$ and 620 $\mathrm{cm}^{-1}$ of the benzene ring. 
Figure. $2 \mathrm{~b}$ shows the atomic concentration of oxygen and nitrogen contained by CS, GQDs, and prGQDs. The oxygen content of GQDs declined in comparison to CS as a result of losing the amorphous structures. prGQDs have a further reduction in oxygen contained with the presence of nitrogen content due to the functionalization in which oxygen functional groups are consumed. Through peak fitting procedure, bonding types including $\mathrm{C}=\mathrm{C} \quad(284.5 \mathrm{eV}), \quad \mathrm{C}-\mathrm{C} \quad(285.17 \mathrm{eV}), \quad \mathrm{C}-\mathrm{O}$ $(286.35 \mathrm{eV}$, for both hydroxyl and epoxy), $\mathrm{C}=\mathrm{O}(287.9 \mathrm{eV})$ and COOH $(288.8 \mathrm{eV})$ are identified from C1s of CS, GQDs and prGQDs(Fig.2c and Fig. S3, and the specific atomic assignment is available in the Supporting Information). Compared to those of the CS and GQDs, there is a new peak arising at $285.7 \mathrm{ev}$ in the $\mathrm{C} 1 \mathrm{~s}$ spectrum of prGQDs owing to the carbon and nitrogen bonding. This bonding state could be further specified via the N1s peak fitting as shown in Figure 2d, where two types of bonding were identified, namely $\mathrm{NH}-\mathrm{C}=\mathrm{O}$ and $\mathrm{C}-\mathrm{NH}$. The former is due to the amidation between $-\mathrm{NH}_{2}$ and $-\mathrm{COOH}$, and the latter is the result of esterification between $-\mathrm{NH}_{2}$ and $\mathrm{OH}$ group. In terms of bonding percentage, the quantity of $\mathrm{NH}-\mathrm{C}=\mathrm{O}$ bond $(80.7 \%)$ is 4 times as much as that of $-\mathrm{NH}_{2}-\mathrm{C}(19.3 \%)$, which suggests that aniline preferably reacts with carboxyl groups on the edge of GQDs. The schematic in Figure 3 summarises the possible main reactions taken place during the one-pot hydrothermal synthesis of prGQDs, namely, upon release, the CS crystallites reacted with aniline via their edge oxygen-containing functional groups, achieving replacement of $-\mathrm{COOH}$ and $-\mathrm{OH}$ with carbon rings.

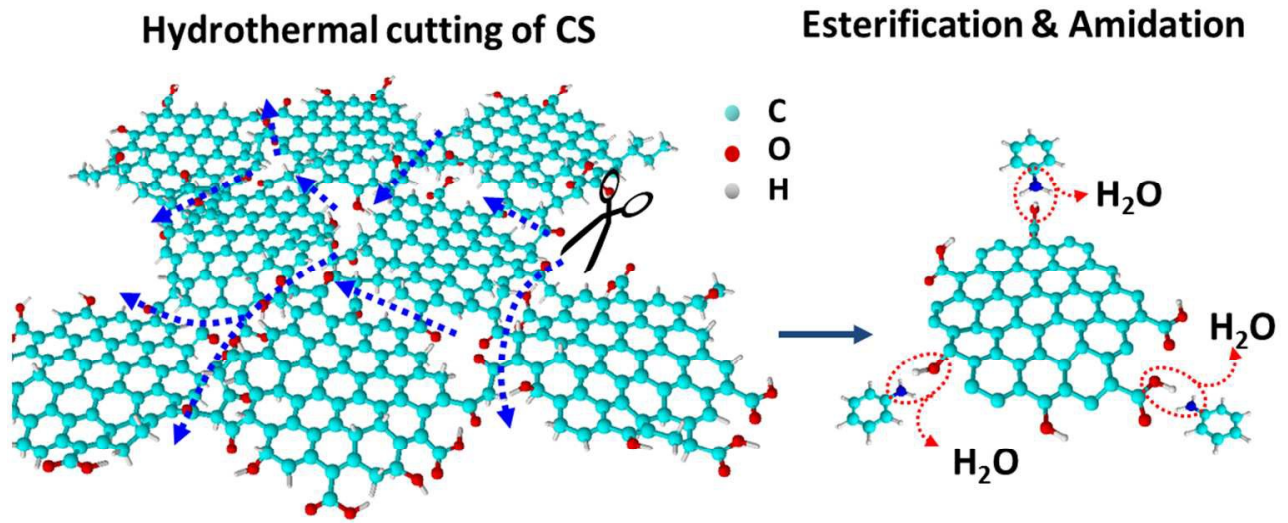

Figure 3. Schematic representation of the process of edge modification by ring structures.

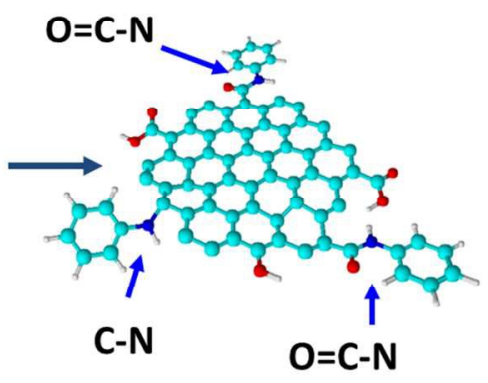

\subsection{Optical properties}

Figure.4a shows the normalized UV/Vis spectra of aqueous dispersions of aniline, CS, GQDs and prGQDs. Upon functionalization with aniline, in contrast to the CS and GQDs, a noticeable shoulder band around $232 \mathrm{~nm}$ arose on the UV/Vis spectrum of prGQDs. It is known that aniline has a primary band at $230 \mathrm{~nm}$ and a secondary band at around $285 \mathrm{~nm}$ in the middle UV region of its UV/Vis spectrum due to aromatic $\pi-\pi^{*}$ transitions of the benzene $\operatorname{ring}^{23}$ as shown in Figure.2a. Therefore, the shoulder band around $232 \mathrm{~nm}$ on the UV/Vis spectrum of prGQDs is due to the primary $\pi-\pi^{*}$ transition of the attached benzene rings. However, the covalent bonding between GQDs and aniline molecules caused the disappearance or significantly weakened the secondary band of the attached benzene ring, therefore, no noticeable secondary band appeared on the UV/Vis spectrum of prGQDs.

Figure $4 \mathrm{~b}$ shows the PL spectra of the four aqueous dispersions under the excitation of $370 \mathrm{~nm}$ (absorbance of dispersions of CS, GQDs, and prGQD were all tuned to 0.1 at $370 \mathrm{~nm}$; For dispersion of aniline, the reaction concentration $1.79 \mathrm{mg} / \mathrm{ml}$ was adopted). Among the four dispersions tested, aniline dispersion, even with the concentration applied for functionalization, showed a much weaker but narrower PL emission around 420nm. Besides, there is no apparent shoulder peak around $420 \mathrm{~nm}$ in the PL of prGQDs detected. The two observations suggest the influence of any excessive residual aniline molecules to the PL of prGQDs could be well eliminated by the purification procedure applied (i.e. dialysis with 500Da membrane). GQDs, in comparison to CS, displays a similar shape of emission spectrum, however, its PL intensity is higher. It suggests that 1) the emitting components in CS are those crystallites which are released to be free GQDs via hydrothermal treatment; 2) The excited electron of those crystallites in CS could be directed to the nonraddiative sites within the amorphous network ${ }^{22}$. Comparing the PL spectra of CS, GQDs and prGQDs, not only has the PL intensity of prGQDs further increased, but also a blue shift of approximately $30 \mathrm{~nm}$ is observed in contrast to CS and GQDs. Such a blue shift might be caused by the reduction in size ${ }^{3-6}$ of GQDs, however, there is no detectable particle size difference from GQDs to prGQDs, the altered UV/Vis and PL behaviors indicate that the aniline functionalization has modified the electronic structures of prGQDs, which may be related to the removal of oxygen functional groups (i.e. - $\mathrm{COOH}$ and $-\mathrm{OH}$ ) and addition of aromatic ring terminals. It has been reported that the PL of GQDs are determined by two types of emissions, namely the intrinsic state emission which possesses size dependence (i.e. quantum confinement), and the defects state emission which is related to the oxygen functional groups $^{20,24,25}$. The aniline conjugation removes the $-\mathrm{COOH}$ and $-\mathrm{OH}$ moieties, hence, the defect state emission (i.e. emission at $471 \mathrm{~nm}$ as marked in Fig. $4 \mathrm{~b}$ ) is suppressed, whilst the intrinsic state emission (i.e. emission at $441 \mathrm{~nm}$ as marked in Fig.4b) is promoted. This leads to a PL blue shift of prGQDs in relation to GQDs.

Overall, both UV/vis absorbance and PL emission analysis demonstrate that the aniline molecules have been conjugated on the edge of GQDs, forming prGQDs with enhanced PL intensity and altered electronic structure.

3.4 PL quenching due to localized $\pi-\pi$ interactions with aromatic molecules at the edge of prGQDs and its application for phenol sensing

To test the PL response of GQDs and prGQDs particles to 


\section{ARTICLE}
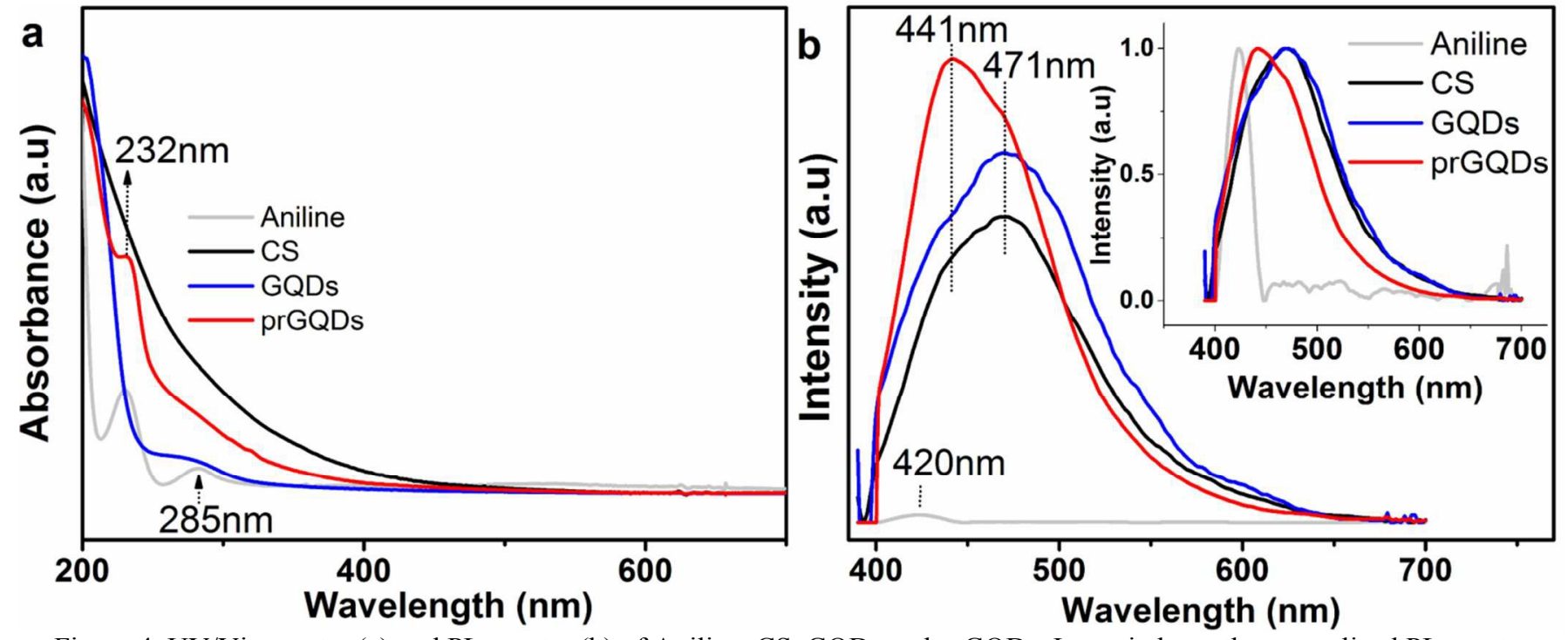

Figure 4. UV/Vis spectra (a) and PL spectra (b) of Aniline, CS, GQDs and prGQDs, Insert in b are the normalized PL spectra

aromatic species, three frequently encountered aromatic molecules were selected, namely benzene, toluene and phenol. Figure $4 \mathrm{a}$ and $\mathrm{b}$ present the observed PL intensity quenching of both GQDs and prGQDs by these three molecules. In the case of GQDs, no effective quenching of PL by any of the three aromatic molecules was observed. In contrast, the PL of prGQDs was quenched by all three aromatic molecules. A possible explanation for the different quenching behaviors of GQDs and prGQDs by aromatic molecules is that ring termination is able to induce $\pi-\pi$ interaction localized at the edge of GQDs. As shown by the HRTEM in Figure 1, GQDs have fine in-plane crystalline $\mathrm{sp}^{2}$ structure. Therefore, GQDs should serve as an excellent platform for $\pi-\pi$ interaction with aromatic molecules. The absence of expected PL quenching suggests two possibilities: a). there are no or little in-plane $\pi-\pi$ interactions between GQDs and those aromatic molecules; $b$ ). if there are in plane $\pi-\pi$ interactions, it does not induce any significant perturbation to the overall electronic structure of GQDs. Considering PL of prGQDs could be quenched, the later rather than the former might make more sense. Specifically, it has been shown by both theoretical and experimental works the edge of GQDs plays a significant role in their physical and chemical properties ${ }^{7-9}$. Therefore, it is highly likely that the ring terminals of prGQDs are able to establish $\pi-\pi$ interactions localized at the edges with aromatic analytes thereby enabling PL quenching through corresponding charge transfer. Such edge-localized $\pi-\pi$ interactions are far more influential to the PL of GQDs than the in plane $\pi-\pi$ interactions. For GQDs, such edge-localized $\pi-\pi$ interactions are significantly disrupted by the presence of abundant oxygen functional groups at the edges (Table S2) which explains their absence of effective PL quenching by aromatic molecules. This also explains why previously the demonstration of aromatic species sensing was scarce in contrast to the popularity of sensing of ionic species by chemically derived GQDs.
Moreover, among the three types of aromatic molecules, phenol appears to be the most efficient quencher. This suggests that the affinity match between aromatic molecules and the linking element nitrogen (between externally introduced ring and edges of GQDs) also plays an important role in determining the efficiency of edge-localized $\pi-\pi$ interaction. In the case of phenol, it appears that the affinity between the hydroxyl of phenol and the nitrogen of prGQDs introduced by aniline functionalization facilitates the edge-localized $\pi-\pi$ interaction, thereby rendering phenol the most efficient edge-localized $\pi-\pi$ interaction, resulting in its highest efficiency in PL quenching(Figure 4c) among the chosen aromatic molecules. On the other hand, benzene and toluene do not possess such a 'binding facilitator' with the nitrogen in the amide group of prGQDs, resulting in less efficient edge-localized $\pi-\pi$ interactions on the edge of prGQDs. Further comparisons against similar aromatic molecules possessing oxygen functional groups including benzoic acid, 4-hydroxybenzonic acid and benzyl alcohol (Figure S5), show that the prGQDs have the highest affinity to phenol, likely due to the combined effect of electrostatic interactions and spatial compatibility. The order of quenching efficiency is phenol $>4$-hydroxybenzoic acid $>$ benzyl alcohol $>$ benzoic acid.

Such a binding selectivity is vital for sensing applications. Figure $4 \mathrm{~d}$ shows the Stern-Volmer plot of the prGQDs, which is a correlation between the PL quenching efficiency and phenol loading. The linear relationship indicates the feasibility of adopting the as-synthesized prGQDs for sensing of phenol, an important pollutant and reaction intermediate. Our phenol sensing test with prGQDs shows that the prGQDs in this study has a detection limit around $10 \mu \mathrm{mol} / \mathrm{L}$ (or equivalent of $1 \mathrm{ppm}$, see supporting information for details) and a linear sensible range of $10 \mu \mathrm{mol} / \mathrm{L} \sim 100 \mu \mathrm{mol} / \mathrm{L}$. 


\section{ARTICLE}
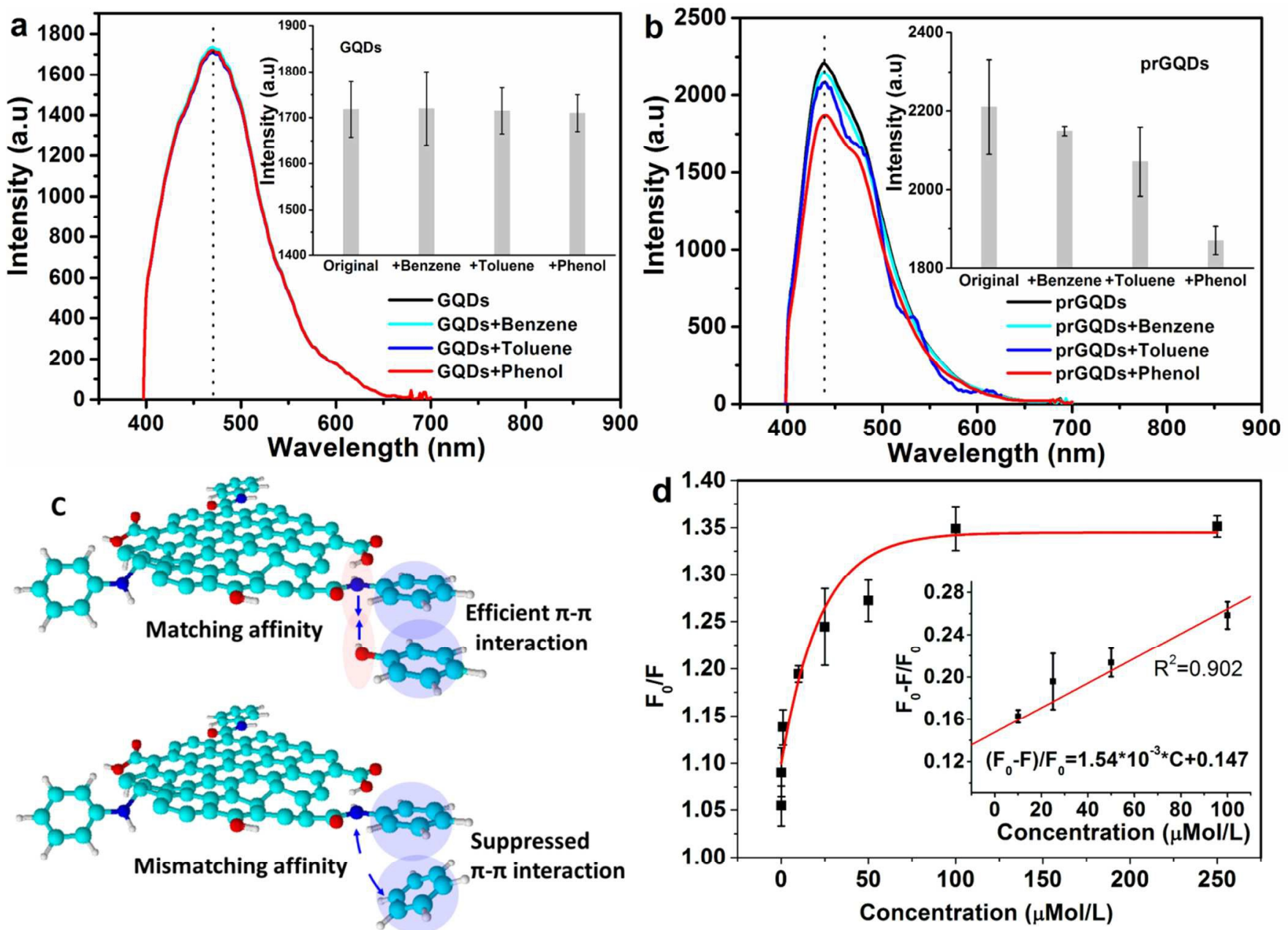

Figure 5. PL intensity quenching tests of GQDs (a) and prGQDs (b) by adding of aromatic molecules (100 $\mu \mathrm{mol} / \mathrm{L})$, Inserts in a and $\mathrm{b}$ are the peak intensities of PL spectra of the tested samples (each error bar is based on measurement of 5 samples); A schematic representation of efficient localized $\pi-\pi$ interaction facilitated by the linking element nitrogen between prGQDs and phenol (c); Stern-Volmer plot of prGQDs with increasing concentration of phenol, Insert is the calibration curve which could be used for sensing of phenol with prGQDs (d).

\section{Conclusions}

In conclusion, we have demonstrated a facile route combining bottom up and top down steps to synthesize the partially ringterminated GQDs (prGQDs). Unlike the regular GQDs, the PL of the prGQDs can be quenched by the aromatic molecules due to the edge-localized $\pi-\pi$ interaction, which could be utilized for facile optical sensing of aromatic hydrocarbons. The selectivity to different aromatic molecules may be tailored through varying the linking element in the functionalization molecules. Additionally, a blue shift of PL was induced by the ring termination, indicating a suppression of defect state emission and promotion of intrinsic state emission. This study also provides insight to the superiority of edge-localized $\pi-\pi$ interaction in perturbing the electronic structure of GQDs over the in-plane $\pi-\pi$ interactions.

\section{Acknowledgements}

S.W. is grateful to the support of a Griffith International Research Postgraduate Scholarship and a CSIRO OCE top-up scholarship. Q.L. wishes to thank a Griffith University Research Infrastructure Grant. The authors thank the Centre for Microscopy and Microanalysis at the University of Queensland for the characterization facilities and support.

\section{Notes}

${ }^{a}$ Environmental Engineering \& Queensland Miro- and Nanotechnology Centre, Griffith University, Nathan, QLD 4111, Australia 
${ }^{b}$ CSIRO Materials Science and Engineering, Gate 5, Normanby Road, Clayton, VIC 3168, Australia

Electronic Supplementary Information (ESI) available: [Morphology of CS, photoluminescence, quenching test, phenol sensing and corresponding discussion]. See DOI: 10.1039/b000000x/

\section{References}

1. K. P. Loh, Q. L. Bao, G. Eda and M. Chhowalla, Nat Chem, 2010, 2, 1015-1024.

2. L. L. Li, G. H. Wu, G. H. Yang, J. Peng, J. W. Zhao and J. J. Zhu, Nanoscale, 2013, 5, 4015-4039.

3. T. Espinosa-Ortega, I. A. Luk'yanchuk and Y. G. Rubo, Physical Review B, 2013, 87.

4. B. Mandal, S. Sarkar and P. Sarkar, Journal of Nanoparticle Research, 2012, 14.

5. M. Zarenia, A. Chaves, G. A. Farias and F. M. Peeters, Physical Review B, 2011, 84.

6. Z. Z. Zhang and K. Chang, Physical Review B, 2008, 77.

7. K. Nakada, M. Fujita, G. Dresselhaus and M. S. Dresselhaus, Physical Review B, 1996, 54, 17954-17961.

8. P. Potasz, A. D. Güçlü and P. Hawrylak, Physical Review B, 2010,81 .

9. K. A. Ritter and J. W. Lyding, Nat Mater, 2009, 8, 235-242.

10. Y. Li, Y. Zhao, H. Cheng, Y. Hu, G. Shi, L. Dai and L. Qu, Journal of the American Chemical Society, 2012, 134, 15-18.

11. C. Hu, Y. Liu, Y. Yang, J. Cui, Z. Huang, Y. Wang, L. Yang, H. Wang, Y. Xiao and J. Rong, Journal of Materials Chemistry B, 2013, 1, 39.

12. Q. Feng, Q. Cao, M. Li, F. Liu, N. Tang and Y. Du, Applied Physics Letters, 2013, 102, 013111.

13. H. Tetsuka, R. Asahi, A. Nagoya, K. Okamoto, I. Tajima, R. Ohta and A. Okamoto, Advanced Materials, 2012, 24, 5333-5338.

14. H. Sun, N. Gao, L. Wu, J. Ren, W. Wei and X. Qu, Chemistry - A European Journal, 2013, 19, 13362-13368.

15. J. R. Lakowicz, Principles of Fluorescence Spectroscopy, Springer, New York, 3rd edn., 2006, ch. 4, pp. 331-348.

16. F. X. Wang, Z. Y. Gu, W. Lei, W. J. Wang, X. F. Xia and Q. L. Hao, Sensor Actuat B-Chem, 2014, 190, 516-522.

17. S. J. Wang, Z. G. Chen, I. S. Cole and L. Qin, Carbon, 2015, 82.

18. L. S. Fan, Y. W. Hu, X. Wang, L. L. Zhang, F. H. Li, D. X. Han, Z. G. Li, Q. X. Zhang, Z. X. Wang and L. Niu, Talanta, 2012, 101, 192-197.

19. D. Pan, J. Zhang, Z. Li and M. Wu, Advanced Materials, 2010, 22, 734-738.

$20 . \quad$ S. J. Zhu, J. H. Zhang, S. J. Tang, C. Y. Qiao, L. Wang, H. Y. Wang, X. Liu, B. Li, Y. F. Li, W. L. Yu, X. F. Wang, H. C. Sun and B. Yang, Adv Funct Mater, 2012, 22, 4732-4740.

21. Y. Q. Dong, J. W. Shao, C. Q. Chen, H. Li, R. X. Wang, Y. W. Chi, X. M. Lin and G. N. Chen, Carbon, 2012, 50, 4738-4743.

22. G. Eda, Y. Y. Lin, C. Mattevi, H. Yamaguchi, H. A. Chen, I. S. Chen, C. W. Chen and M. Chhowalla, Adv Mater, 2010, 22, 505509.

23. C. W. N. Cumper and A. Singleton, Journal of the Chemical Society B, 1968, 649-651.

24. F. Liu, M. H. Jang, H. D. Ha, J. H. Kim, Y. H. Cho and T. S. Seo, Advanced Materials, 2013, 25, 3657-3662.

25. J. Wei and J. Qiu, Advanced Engineering Materials, 2015, 17, 138-142. 

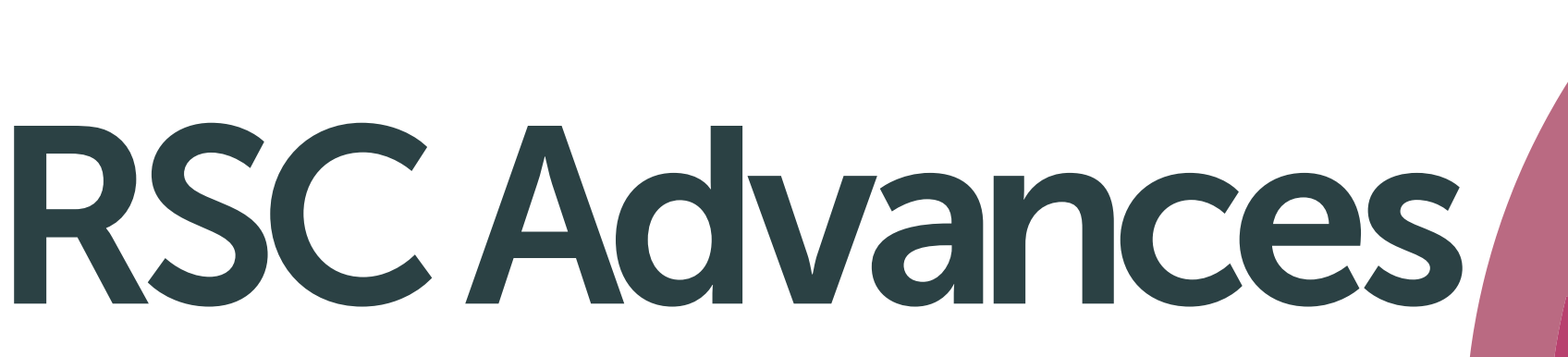

Volume1 Number1 Jan 2013 $\mid$ Pages 1-100

\section{RSC Advances}

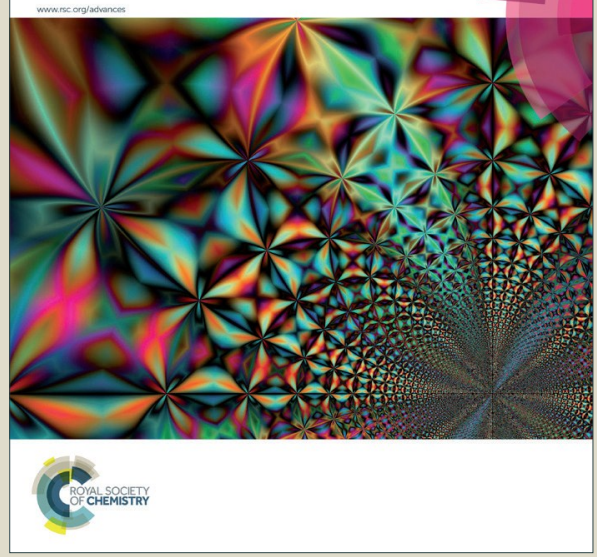

This is an Accepted Manuscript, which has been through the Royal Society of Chemistry peer review process and has been accepted for publication.

Accepted Manuscripts are published online shortly after acceptance, before technical editing, formatting and proof reading. Using this free service, authors can make their results available to the community, in citable form, before we publish the edited article. This Accepted Manuscript will be replaced by the edited, formatted and paginated article as soon as this is available.

You can find more information about Accepted Manuscripts in the Information for Authors.

Please note that technical editing may introduce minor changes to the text and/or graphics, which may alter content. The journal's standard Terms \& Conditions and the Ethical guidelines still apply. In no event shall the Royal Society of Chemistry be held responsible for any errors or omissions in this Accepted Manuscript or any consequences arising from the use of any information it contains. 